\title{
Haploidentical Stem Cell Transplantation With Post- Transplantation Cyclophosphamide for Aggressive Lymphomas: How Far Have We Come and Where Are We Going?
}

\author{
Dilan A. Patel
}

\begin{abstract}
Haploidentical hematopoietic stem cell transplantation (haplo-HSCT) with post-transplant cyclophosphamide (PTCy) offers universal donor availability and can potentially cure relapsed or primary refractory Hodgkin lymphoma (HL) and non-Hodgkin lymphoma (NHL). However, a conditioning regimen intensity that balances the graftversus-lymphoma (GvL) effect with regimen-related toxicities (RRTs) has not yet been optimized. Limited data exist on the management of relapse, which is common post-transplant. Few prospective or randomized control trials have been conducted on lymphoma patients undergoing haplo-HSCT. Therefore, the current review aims to summarize published retrospective data in the field to help guide clinical decision making for high-risk patients. Retrospective studies in the field are characterized by variability in patient population and sample sizes, eligibility criteria, number of prior treatments (e.g., chemotherapy, radiation therapy, and autologous transplant), graft source (bone marrow or peripheral blood), as well as choice and intensity of the conditioning regimen (non-myeloablative, reduced intensity, or myeloablative). Nonetheless, common themes that emerge from the literature include: 1) Enhanced donor availability and selection with haplo-HSCT with success in heterogeneous patient populations; 2) Outcomes that are comparable if not superior to matched related (MRD) or unrelated (MUD) donor transplants; 3) The benefit of PTCy for reducing incidence of relapse and chronic graft-versushost disease (GvHD); 4) Presence of co-morbidities leading to poorer transplant-related outcomes; and 5) The need for novel approaches to address disease relapse, particularly for patients with active disease at the time of transplant. Excellent transplant-related outcomes with haplo-HSCT with PTCy have been seen for HL and NHL based on retrospective data. Further studies are needed to determine integration with advanced cellular therapy techniques, such as chimeric antigen receptor (CAR) T-cell, antibody drug conjugates, and checkpoint inhibitors. Graft manipulation may be another avenue for future
\end{abstract}

Manuscript submitted October 17, 2018, accepted December 14, 2018

Vanderbilt Ingram Cancer Center, Vanderbilt University School of Medicine, 2220 Pierce Avenue, Nashville, TN 37232, USA.

Email: Dilan.a.patel@gmail.com

doi: https://doi.org/10.14740/wjon1164 research.

Keywords: Haploidentical hematopoietic stem cell transplantation; Post-transplant cyclophosphamide; Aggressive lymphoma

\section{Introduction}

Patients with relapsed/primary refractory $(\mathrm{R} / \mathrm{R})$ aggressive or transformed indolent lymphomas have a poor prognosis and are often referred for consideration of allogeneic hematopoietic stem cell transplantation (HSCT) with or without advanced cellular therapy [1-3]. Autologous stem cell transplantation (ASCT) with preceding high doses of combination chemotherapy is the next step in management of patients with Hodgkin lymphoma (HL), or various subtypes of non-Hodgkin lymphoma (NHL) who relapse after induction therapy and have chemotherapy-sensitive disease $[4,5]$. Many patients, however, with continued disease burden or chemotherapy-refractory disease require HSCT, which is potentially curative. Undergoing HSCT is limited by cost, donor availability, particularly for racial and ethnic minorities, and logistical delays for unrelated donors, with disease progression potentially occurring during the search process $[1,2,6]$. Alternative donor transplantation platforms have been developed to address these concerns.

Haploidentical hematopoietic stem cell transplantation (haplo-HSCT) is increasingly used for patients with high-risk hematologic malignancies (HMs) due to near universal donor availability from first- and even second-degree relatives as well as outcomes that are similar to matched related (MRD) and unrelated (MUD) donors [7-10]. Historically, haplo-HSCT with T-cell-replete (TCR) grafts has been limited due to severe, acute and chronic graft-versus-host disease (GvHD) and graft rejection resulting from intense bidirectional alloreactivity in the setting of human leukocyte antigen (HLA) mismatches, with unacceptably high rates of non-relapse mortality (NRM) [11-13]. The platform was essentially abandoned until the mid-1990s when Aversa and colleagues at the University of Perugia, Italy developed the concept of T-cell depletion (TCD) with CD34 stem cell positive selection and "megadose" infusion. This concept resulted in high rates of engraftment and 
acceptable rates of GvHD $[12,13]$. However, problems with delayed immune reconstitution (IR) and resultant increased risk of opportunistic infections and relapse spawned more sophisticated means of graft manipulation along with changes to the conditioning regimen or post-transplant immune suppression $[12,13]$. Furthermore, TCD requires extensive technical expertise and is limited by cost and reproducibility. As a result, its availability is limited to only a small portion of cancer centers throughout the world $[12,13]$.

The use of high doses of post-transplant cyclophosphamide (PTCy) has transformed the field of haplo-HSCT by allowing the successful use of TCR grafts [11, 14-17]. Researchers at Johns Hopkins University (JHU) have made groundbreaking strides in facilitating drug-induced immunologic tolerance with cyclophosphamide since the 1960s [17-19]. Alloreactive T-cells, which make up 1-10\% of cells in grafts and are responsible for graft rejection and GvHD, are differentially sensitive to high doses of cyclophosphamide due to the lack of expression of the enzyme aldehyde dehydrogenase (ALDH) [11, 2022]. On the other hand, hematopoietic stem cells (HSCs) and progenitor cells, along with T-regulatory cells that promote IR and reduce GvHD, are able to metabolize cyclophosphamide due to ALDH expression, allowing for selective deletion of rapidly dividing alloreactive T-cells after transplantation [11, $22,23]$.

Further research at JHU in 2002 showed improvements in GvHD and engraftment with non-myeloablative conditioning (NMA) regimens using cyclophosphamide, fludarabine, and low-dose total body irradiation (TBI) along with PTCy in humans $[16,17]$. The phase 1 clinical trial included 13 patients with high-risk HM, one of whom had refractory diffuse mixed small and large cell lymphoma. It showed the potential for sustained engraftment with non-severe acute GvHD [16]. Thereafter, Luznik and colleagues at JHU and the Fred Hutchinson Cancer Research Center published their results on a trial investigating the safety and efficacy of PTCy after similar NMA conditioning and TCR haplo-HSCT for 68 high-risk HM patients, including 13 with HL and 10 with NHL $[16,17]$. The study showed a cumulative NRM and relapse at 1 year of $15 \%$ and $51 \%$, respectively, with cumulative incidence of grades II-IV and III-IV acute GvHD at day 200 of 34\% and $6 \%$, respectively. Importantly, patients with lymphoid malignancies were found to have a significantly improved event-free survival (EFS) compared to those with myeloid malignancies $(\mathrm{P}=0.02)$, suggesting an inherent graft-versus-lymphoma (GvL) effect $[16,17]$. These studies provided the basis for further investigation into the curative potential of haplo-HSCT for lymphomas [24].

\section{Comparison of Donor Platforms for R/R HL}

HSCT offers curative potential for patients with HL that has progressed despite high-dose chemotherapy and ASCT [2, 5, 25]. Initial attempts with myeloablative conditioning (MAC) regimens for improved disease control followed by HSCT, however, were plagued by unacceptably high NRM of up to $50 \%$ and persistent relapse, with few patients being cured long-term [25-27]. NMA regimens were subsequently investigated, with encouraging results in terms of treatment-related mortality (TRM) [28-31]. Accordingly, Burroughs and colleagues designed a multi-institutional retrospective study to assess outcomes based on donor cell source for R/R HL patients receiving NMA regimens [24]. The study included 38 MRD, 24 MUD, and 28 haploidentical (haplo) donors who were similar in terms of hematopoietic cell transplantation co-morbidity index (HCT-CI) and heavily pre-treated, with a median of five regimens [24,32]. Additionally, $92 \%$ of patients failed ASCT and $83 \%$ progressed despite local radiation therapy. The results indicate that NRM was significantly lower for haplo recipients compared to matched recipients $(\mathrm{P}=0.02)$. Furthermore, 2-year relapse incidence was significantly lower for haplo recipients compared to MRD or MUD ( $\mathrm{P}=0.01$ and 0.03 , respectively). Overall survival (OS) did not differ between the groups. Interestingly, despite the HLA mismatch, the incidence of acute grades III-IV and extensive chronic GvHD were lower with haplo-HSCT with PTCy, $11 \%$ and $35 \%$, compared to MRD, $16 \%$ and $50 \%$, and MUD, $8 \%$ and $63 \%$, respectively, though these differences were not statistically significant. One potential confounding factor was the use of bone marrow (BM) allografts for haplo-HSCT recipients compared to matched donors who received peripheral blood stem cells (PBSCs), which have up to 10-log higher T-cells and are associated with chronic GvHD [11,24]. Regardless, the study provided evidence that NMA regimens and haplo-HSCT with PTCy can result in favorable NRM and transplant-related outcomes [33]. Still, disease relapse was noted to be problematic (Table 1) [34-45].

Haplo-HSCT with PTCy is hypothesized to offer a greater GvL effect based on HLA disparity [33]. Mariotti and colleagues in Italy provide further evidence for this effect based on retrospective data, and suggest that haplo-HSCT may offer more benefit than matched sibling donor (MSD) even for patients with available donors [34]. A total of 64 patients were included, with 34 undergoing MSD and 30 undergoing haplo transplant [34]. At a median follow-up of 46 months, the results indicate significantly improved neutrophil and platelet engraftment in the MSD versus haplo-HSCT cohort (14 days vs. 19 and 11 days vs. $23, \mathrm{P}<0.005$, respectively). However, the patients who underwent haploidentical transplant had significantly reduced 3-year cumulative relapse and chronic GvHD (13\% vs. $62 \%, \mathrm{P}=0.0001$ and $3 \%$ vs. $32 \%, \mathrm{P}=0.003$, respectively), with resultant improved progression-free survival (PFS) $(60 \%$ vs. $29 \%, \mathrm{P}=0.04)$. OS and NRM did not differ significantly between the groups. Multi-variate analysis showed that patients receiving a haplo transplant (hazard ratio (HR) $0.17, \mathrm{P}=0.02$ ) and achieving optimal disease control with complete remission (CR) before HSCT (HR 0.6, P < 0.0001) were the only variables independently associated with reduced risk of relapse. Moreover, relapse was lower even after matching the two cohorts in terms of pre-transplant disease status or graft type. The findings provide additional evidence about the necessity of optimizing disease control prior to HSCT, as no patients who were in CR and only $10 \%$ of patients in partial response (PR) before transplant relapsed, with 3-year OS of $90 \%$ and $60 \%$, respectively [34].

Given the accumulating evidence for haplo-HSCT for 
Table 1. : Outcomes From Multiple Retrospective Studies on NHL and HL Patients Receiving Haplo-HSCT and PTCy for GvHD Prophylaxis

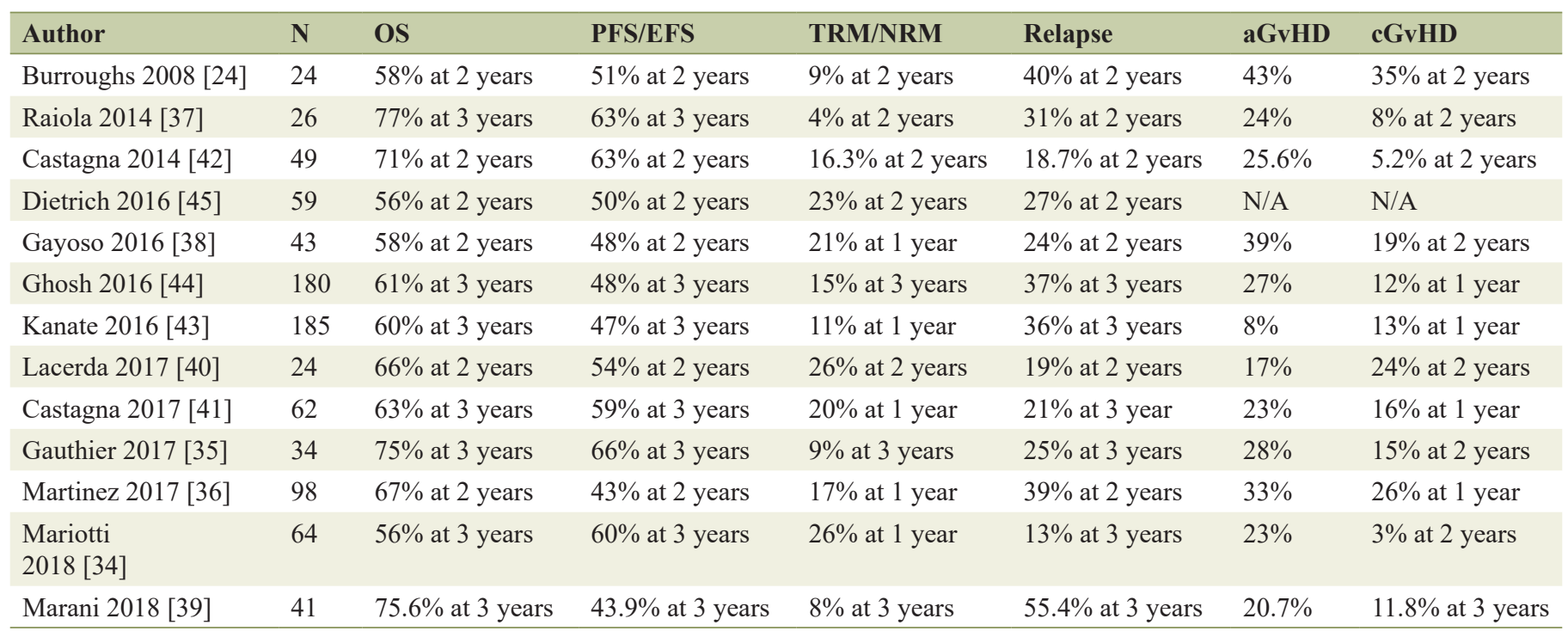

Author: last name of the first author and year of publication in a chronological order; $\mathrm{N}$ : the total number of haplo patients in the study; OS: overall survival at time point; PFS: progression-free survival/event-free survival at time point; TRM/NRM: transplant-related mortality/non-relapse mortality at time point; relapse: percentage of patients having relapse at time point; aGvHD: acute graft-versus-host disease, percentage of patients having grades II-IV disease at 100 days post-transplant; cGvHD: chronic graft-versus-host disease, combined limited and extensive.

R/R HL, Gauthier and colleagues in France conducted a retrospective study comparing patients receiving haplo grafts $(\mathrm{n}=34)$ to mismatched unrelated donors (MMUDs) $(\mathrm{n}=37)$ or cord blood $(\mathrm{CB})$ grafts $(\mathrm{n}=27)$ [35]. After multi-variate analysis with adjustment for co-variates, MMUD and CB patients were found to have a significantly lower GvHD relapsefree survival (GRFS) than haplo-HSCT patients (HR 2.02, P $=0.03$, and HR 2.43, P $=0.009$, respectively). The incidence of chronic GvHD was significantly lower in the haplo group compared to MMUD and CB (15\% vs. $48 \%$ vs. $39 \%$, respectively, $\mathrm{P}=0.006)$. Notably, however, the haplo-HSCT cohort was less heavily pre-treated, as evidenced by $23 \%$ who did not undergo prior ASCT, which may potentially explain the better outcomes. Other transplant-related outcomes such as OS, EFS, relapse, and NRM did not significantly differ between the groups. Consistent with other studies, disease status at the time of transplant was the only factor that significantly influenced OS, relapse, and EFS. Regardless, however, the data suggest consistent benefit with PTCy for chronic GvHD incidence [35].

Most recently, Martinez and a multi-national group conducted a retrospective study in 2017 on 709 patients with R/R HL to determine optimal donor source between haplo-HSCT with PTCy compared to MRD and MUD [36]. The analysis included 98 patients who received haplo-HSCT, 338 MRD, and 273 MUD. For haplo patients, BM was the graft source in $60(61 \%)$ and PBSC in $38(39 \%)$ of the patients. At a median follow-up time of 29 months, the results indicate that haploHSCT is associated with significantly lower chronic GvHD compared to MUD (26\% vs. $41 \%, \mathrm{P}=0.04)$, which is consistent with multiple prior studies [24, 34, 35]. Grades II-IV acute GvHD, however, were higher in the haplo group compared to
MRD (33\% vs. $18 \%, \mathrm{P}=0.003)$. No other differences in transplant-related outcomes were noted between the groups, including NRM, relapse, or progression [36]. The study reflects the growing use of PBSC as a graft source, which may explain the higher acute GvHD incidence seen in the study [36].

\section{Application of Haplo-HSCT for HL}

The improved outcomes in haplo-HSCT recipients with reduced incidences of GvHD are hypothesized to be from the immune modulating properties of high-dose PTCy. Accordingly, additional retrospective analyses were performed to replicate these outcomes and to investigate the purported greater GvL effect from HLA disparity. Raiola and colleagues from Milan, Italy, conducted a retrospective analysis of 26 patients with R/R HL, all of whom had prior ASCT and $65 \%$ of whom had active disease at the time of transplant [37]. All the patients received the JHU derived NMA regimen with unmanipulated BM grafts $[16,17,37]$. The patients were young, with a median age of 31 , and were otherwise healthy based on a low HCT-CI. The results showed sustained donor cell engraftment in 25/26 patients. The incidence of grades II-IV acute GvHD and chronic GvHD was $24 \%$ and $8 \%$, respectively. At a median follow-up of 2 years, 21 patients were alive, 20 of whom were disease free. The actuarial 3-year OS and PFS were 77\% and $63 \%$, respectively. TRM and relapse occurred in $4 \%$ and $31 \%$ of patients, respectively. Relapse occurred at a median of 5 months post-transplant, more so in heavily pre-treated and chemotherapy-refractory cases. Two patients were considered chemotherapy and radiation therapy refractory, and both died from disease progression. The implications of the study are 
three-fold: 1) Showing generalizability of the JHU protocol; 2) Providing further evidence for NMA over MAC regimens; and 3) Stimulating further research efforts into the factors that influence disease relapse.

In order to investigate factors contributing to post-transplant relapse, Gayoso and colleagues in Spain conducted a retrospective multi-institutional study to investigate busulfan rather than TBI as part of a reduced intensity conditioning (RIC) regimen [38]. The study included 43 patients, $72 \%$ of whom received PBSC grafts, unlike in the aforementioned Raiola study $[37,38]$. The results showed that $42 / 43$ patients engrafted, with cumulative incidence of grades II-IV acute GvHD and chronic GvHD of 39\% and 19\%, respectively, though immune suppression therapy was discontinued earlier to promote IR and reduce relapse. At a median follow-up of 25.5 months, $27 / 43$ were alive, 22 of whom were disease free. The cumulative incidence of 1 year NRM and relapse at 2 years was $21 \%$ and $24 \%$, respectively. The estimated 2 -year PFS and OS was $48 \%$ and $58 \%$, respectively. CR prior to transplant correlated with significantly better EFS and OS compared to patients with evidence of disease $(78.5 \%$ vs. $33.5 \%, \mathrm{P}=0.015$, and $86 \%$ vs. $46 \%, P=0.044$, respectively). Importantly, no differences were found for transplant-related outcomes such as EFS, OS, NRM, and acute and chronic GvHD between PBSC and BM graft sources, suggesting that PBSC grafts can be safely used [38]. The higher NRM and acute and chronic GvHD rates seen in this series, however, may reflect a tradeoff that exists with early tapering of immune suppression to reduce relapse, indicating the need for more innovative approaches for prevention and treatment.

Marani and colleagues retrospectively analyzed 41 patients to determine the risk factors for post-transplant relapse using the HCT-CI along with PET/CT findings [32, 39]. Multiple patient and disease variables were assessed, including refractoriness of the disease, relapse within 6 months from ASCT, amount of pre-transplant chemotherapy received, sex mismatch, tumor burden, and HCT-CI along with PET/CT findings. The results showed successful engraftment in 40/41 patients. Low rates of severe, acute, and moderate to severe chronic GvHD of $2.4 \%$ and $11.8 \%$, respectively, were seen, along with 3-year OS, PFS, and GFRS rates of 75.6\%, 43.9\%, and $39 \%$, respectively. As expected, multi-variate analysis showed that a HCT-CI greater than 3 predicted a lower 3-year relapse rate, PFS, and GRFS, along with higher NRM and OS. The overall findings provide evidence that patients with poor performance status and multiple co-morbidities fare poorly with haplo-HSCT, even those with potentially curable diseases, emphasizing the importance of careful patient selection given the inherent morbidity and mortality associated with HSCT.

An advantage of haplo-HSCT with PTCy is donor availability and reproducibility, as shown by Lacerda and colleagues, who retrospectively reviewed outcomes of 24 patients who received RIC regimens in six low resources centers in Brazil [40]. The patients had a median age at transplant of 27. Thirteen $(54 \%)$ received unmanipulated BM while 11 (46\%) received granulocyte colony-stimulating factor mobilized PBSC grafts. Twenty-two patients had a prior ASCT, suggestive of biologically aggressive disease. The patients were extensively pre-treated, with 10 in second $\mathrm{CR}$, three in third CR, nine in
PR, and two with refractory disease. At a median follow-up of 2 years, the cumulative incidence of NRM was $26 \%$ and rates grades II-IV acute GvHD and chronic GvHD were 17\% and $24 \%$, respectively. Relapse or progression occurred in $19 \%$ of patients at 2 years. Estimation of PFS and OS at 2 years was $54 \%$ and $66 \%$, respectively. The estimated higher NRM compared to other studies is likely from increased infection risk more so than GvHD, which may be a reflection of health care access and socioeconomic limitations [40]. The results were encouraging, however, showing the generalizability of unmanipulated TCR grafts with PTCy in developing countries.

A retrospective study by Castagna et al in 2017 from centers in France and Italy showed generalizability and favorable outcomes with NMA and RIC regimens along with PTCy. Sixty-two patients were included, with 39 (63\%) receiving $\mathrm{BM}$ and 23 (37\%) receiving PBSC grafts [41]. A nuance existed with the conditioning regimen, however, in that 51 $(89 \%)$ of patients received the conventional JHU-based NMA regimen, while $11(19 \%)$ received thiotepa-based RIC. The results indicated a 3-year OS, PFS, and relapse incidence of $63 \%, 59 \%$, and $21 \%$, respectively. NRM at 1 year was $20 \%$. The cumulative incidences of grades II-IV acute GvHD and chronic GvHD were 23\% and 16\%, respectively. Only $4 \%$ of patients had severe acute GvHD. Notably, patients who were in CR prior to haplo-HSCT had a markedly better 3-year OS and PFS (84\% and 76\%, respectively), compared to patients with stable or progressive disease. A unique finding in this study, while not statistically significant, was the trend towards reduced relapse with $\mathrm{PBSC}$ compared to $\mathrm{BM}$ (HR 0.36, $\mathrm{P}=$ 0.12 ), which warrants further investigation, given the greater number of T-cells in PBSC. The overall findings indicate that active uncontrolled disease status at the time of transplant and HCT-CI over 2 are predictors of higher NRM, suggesting that the potent GvL effect from haplo-HSCT is limited by disease burden in certain patients. These findings highlight the importance of optimal disease debulking prior to transplant along with careful patient selection for transplant based on co-morbidities.

\section{Application of Haplo-HSCT for Both HL and NHL}

Castagna and colleagues at two institutions in Italy collaborated on a retrospective study of 49 advanced HL and NHL patients to assess transplant-related outcomes [42]. The NMA conditioning regimen was based on the JHU protocol, including PTCy. A total of 27 patients had HL and 17 had NHL. The median age at transplant was 45 , with $34 / 49$ (69.3\%) having an HCT-CI of 1 - 2, indicating few co-morbidities. As expected, the patients were heavily pre-treated, with $27 / 49$ (55\%) relapsing after ASCT, 7/49 (14\%) relapsing after HSCT and four $(8.2 \%)$ having refractory disease. The incidences of grades II-IV acute GvHD and chronic GvHD were $25.6 \%$ and 5.2\%, respectively, at a median follow-up of 20.6 months, which are considered comparatively low rates [42]. The projected OS and PFS at 2 years were $71 \%$ and $63 \%$, respectively. In terms of relapse, $18.7 \%$ of patients had histologically confirmed relapse at a median time of 4.4 months. The NRM incidence was 
$16.3 \%$ at 2 years, which is consistent with data from MRD and MUD platforms [42]. The data on infections post-transplant, which were mainly viral, warrant further studies on the effect of PTCy on IR, particularly due to evidence of potentially early delayed reconstitution of T-cells $[11,22]$.

A large, retrospective multi-institutional study designed to compare outcomes for lymphoma patients who received haplo-HSCT with PTCy to MUD transplants with or without anti-thymocyte globulin (ATG) was conducted by Kanate et al in 2016 [43]. A total of 917 patients were included, with 185 haplo and 732 MUD patients, of whom 241 received ATG while 491 did not. Patients received either RIC or NMA regimens. Day 100 cumulative incidence of acute GvHD did not differ between the groups. However, the data indicate that severe acute GvHD was significantly higher in the MUD group, without or with ATG, compared to haplo-HSCT $(\mathrm{P}=0.001$ and $\mathrm{P}=0.01$, respectively), suggesting beneficial effects of PTCy despite the HLA-mismatch. The 1 year chronic GvHD incidence for haplo, MUD without ATG, and MUD with ATG was $13 \%, 51 \%$, and $33 \%$, respectively $(\mathrm{P}<0.001)$. Relapse, progression, or OS at 3 years did not differ significantly between the groups. Multi-variate analysis also showed non-significant differences between the groups for NRM, relapse, or PFS. Deaths due to GvHD were rare in the haplo-HSCT group (1\%), suggesting that even with TCR grafts, PTCy has the ability to mitigate both acute and chronic GvHD risk with comparable disease control to matched transplants, though the results may be confounded with the more widespread use of BM grafts in haploidentical recipients [43]. The results suggest that PTCy should be potentially used in the MUD population and that further studies should investigate the impact of graft source on GvHD in the context of PTCy. This has already been done for acute leukemia, with an analysis by Ruggeri showing higher acute GvHD, though no difference was observed in outcomes with BM versus PBSC grafts [46].

In a Center for International Blood and Marrow Transplant Research analysis, Ghosh and colleagues built on the results of Kanate et al by conducting the largest known study to date comparing lymphoma outcomes with RIC haplo-HSCT with PTCy to gold-standard MSD transplants $[43,44]$. The purpose of the analysis was to assess whether haplo-HSCT may be a better option than MSD as a potential first-line therapy for R/R disease, given the promising data from multiple other studies for reducing chronic GvHD and NRM [24, 36-43]. A total of 987 patients were evaluated, of whom 180 had a haplo donor and 807 had an MSD. Both were treated with RIC regimens. Multiple histologies were included, namely diffuse large Bcell lymphoma, follicular lymphoma, mantle cell lymphoma, mature natural killer/T-cell lymphomas, and HL. Based on disease risk index, patients in the haplo-HSCT group were considered to have a higher risk [44]. At a median follow-up of 3 years, the authors found that platelet engraftment was significantly delayed in the haplo-HSCT group, while neutrophil engraftment was similar. The incidence of grades II-IV acute GvHD was similar at day 100, though chronic GvHD was significantly lower in the haplo-HSCT group at 1 year (12\% vs. $45 \%, \mathrm{P}<0.001)$, with the findings confirmed on multi-variate analysis (relative risk $0.21,95 \%$ confidence interval 0.14 to $0.31, \mathrm{P}<0.001)$. Notably, similar to other haplo-HSCT proto- cols, BM was used as the graft source more than PBSCs [24]. Importantly, the 3-year rates of NRM, relapse or progression, PFS, and OS did not differ significantly between the groups, which was also confirmed on multi-variate analysis. The lower chronic GvHD, shown in multiple other studies, suggests the need to further explore the influence of PTCy on inducing Tcell tolerance $[11,22]$. Based on the study results, additional studies are also needed to investigate the relationship between graft source and PTCy on chronic GvHD, given the trend towards PBSC rather than BM grafts [46]. While retrospective, this study is the first to show comparable outcomes on a large scale between haplo-HSCT and MSD grafts for HL and NHL [44].

In a retrospective study of the European Society for Blood and Marrow Transplantation registry, Dietrich and colleagues investigated outcomes of NHL patients treated with various haplo platforms [45]. Of the 97 patients who met inclusion criteria, 59 received PTCy for GvHD prophylaxis, while the remaining 38 received alternative donor platforms involving either ex vivo or in vivo TCD. The patients were similar in baseline characteristics with the exception of the PTCy group being older and having more recent transplants [45]. Nonetheless, the PTCy group had a significantly better OS and PFS than patients receiving other haplo protocols $(\mathrm{P}=0.0036, \mathrm{HR} 0.39$, and $\mathrm{P}=0.0003$, HR 0.36, respectively). Additionally, 2 year NRM was significantly lower with PTCy compared to other platforms (25\% vs 44\%, p =0.0086, HR 2.9). The authors also conducted a registry analysis of the same patient population to compare haplo-HSCT outcomes with patients who received MRD $(n=2,024)$ and MUD $(n=437)$ transplants. They found similar non-significant differences in OS, NRM, relapse, and acute GvHD, though haplo-HSCT patients were noted to have significantly lower extensive chronic GvHD $(\mathrm{P}=0.057)$, consistent with other studies [24, 36-43]. The results provide further support for the use of PTCy-based GvHD prophylaxis regimens for improving outcomes.

\section{Long-Term Implications}

Studies on haplo-HSCT with PTCy for R/R aggressive lymphomas indicate the importance of adequate disease control prior to transplant. Methods to better debulk the disease include immune checkpoint inhibitors and antibody drug conjugates, though the sequencing of therapy in conjunction with HSCT is still in question [47-51]. While data suggest a more favorable GvL effect with haplo-HSCT compared to matched donors, the presence of active disease may be more than the graft can overcome, leading to poor outcomes and inevitable relapse [45]. It has been suggested also that high doses of PTCy may have an anti-tumor effect, possibly by influencing the BM microenvironment [45].

Novel methods of graft selection and manipulation may be needed to improve outcomes. Recent studies have shown that natural killer $(\mathrm{NK})$ cell immunoglobulin-like receptor-ligand (KIR) mismatches in donor to recipient direction may be associated with an improved GvL effect in patients with HM [52]. Wanquet et al conducted a retrospective study of 144 patients, 
$93(65 \%)$ of whom had lymphoid malignancies, to determine the impact of KIR-ligand mismatches on outcome of patients undergoing TCR haplo-HSCT [52]. A separate analysis was done on patients who were in CR $(n=81)$ or active disease $(n=63)$ at the time of transplant, which revealed that for patients in the latter category with a KIR-ligand mismatch, the risk of relapse was significantly lower $(\mathrm{HR} 0.21, \mathrm{P}=0.013)$ and PFS was significantly higher $(\mathrm{HR} 0.42, \mathrm{P}=0.028)$, with no increase in GvHD or NRM, compared to patients without KIR mismatches [52]. These findings suggest the need for further investigation into NK cell immunotherapy for patients with HL or NHL and active disease at the time of consideration of haplo-HSCT [53-55]. In addition, Aversa and the Perugia group have developed a new method for immune tolerance induction with NMA regimens and CD34 positively selected TCD haplo-HSCT with PTCy for promoting engraftment and reducing rejection, which offers exciting potential for patients with R/R lymphomas [55].

The success of chimeric antigen receptor (CAR) T-cells in the treatment of HM, including aggressive lymphomas, suggests the potential for incorporation into haplo-HSCT platforms [56]. Current management options for relapsed disease, including donor lymphocyte infusions, are associated with a substantial risk of TRM from GvHD, and have not been extensively tested after HSCT. CAR T-cells have the potential to induce remission in these patients, though TRM is still problematic due to GvHD and cytokine release syndrome [56]. Similar to studies on R/R lymphomas with haplo-HSCT and PTCy, therapy with CAR T-cells has been shown to be more efficacious in patients with lower tumor burden, which highlights the need for better detection of minimal residual disease and should prompt investigation into the optimal timing of infusion for prevention and treatment [56].

\section{Conclusions}

The clinical management of aggressive R/R lymphomas involves consideration for haplo-HSCT protocols with PTCy due in part to multi-national studies showing comparable and even improved outcomes in relation to matched donors, particularly for reduced incidence of relapse and chronic GvHD. The majority of these analyses are retrospective in nature, thus necessitating the need for prospective and randomized comparative studies to help determine optimal approaches specific to each patient, including the potential for haplo-HSCT as a front-line option. Studies on IR post-haplo-HSCT may also be warranted to investigate the properties of PTCy on T-cell subsets. Future trials should take into consideration the integration of advanced cellular therapy with CAR T-cells and NK cell immunotherapy with HSCT.

\section{Acknowledgments}

The author thanks mentors, Dr. Nina Wagner-Johnston, and Dr. Adetola Kassim, for continued guidance in the fields of lymphoma and stem cell transplantation, respectively.

\section{Conflict of Interest}

The author states that no conflict of interest exists regarding this review paper.

\section{Abbreviations}

haplo-HSCT: haploidentical hematopoietic stem cell transplantation; PTCy: post-transplant cyclophosphamide; HL: Hodgkin lymphoma; NHL: non-Hodgkin lymphoma; GvL: graft-versus-lymphoma; RRTs: regimen-related toxicities; GvHD: graft-versus-host disease; HSCT: hematopoietic stem cell transplantation; ASCT: autologous stem cell transplantation; R/R: relapsed/primary refractory; MRD: matched related donor; MUD: matched unrelated donor; TCR: T-cell-replete; HLA: human leukocyte antigen; NRM: non-relapse mortality; TCD: T-cell depletion; IR: immune reconstitution; JHU: Johns Hopkins University; ALDH: aldehyde dehydrogenase; HSCs: hematopoietic stem cells; NMA: non-myeloablative conditioning; TBI: total body irradiation; EFS: event-free survival; MAC: myeloablative conditioning; HCT-CI: hematopoietic cell transplantation co-morbidity index; BM: bone marrow; MMUD: mismatched unrelated donor; $\mathrm{CB}$ : cord blood; GRFS: relapse-free survival; OS: overall survival; PBSCs: peripheral blood stem cells; HR: hazard ratio; RIC: reduced intensity conditioning; CR: complete remission; ATG: anti-thymocyte globulin; HM: hematologic malignancy; NK: natural killer; KIR: receptor-ligand; CAR: chimeric antigen receptor

\section{References}

1. van Besien K. Current status of allogeneic transplantation for aggressive non-Hodgkin lymphoma. Curr Opin Oncol. 2011;23(6):681-691.

2. Sarina B, Castagna L, Farina L, Patriarca F, Benedetti F, Carella AM, Falda M, et al. Allogeneic transplantation improves the overall and progression-free survival of Hodgkin lymphoma patients relapsing after autologous transplantation: a retrospective study based on the time of HLA typing and donor availability. Blood. 2010;115(18):3671-3677.

3. Appelbaum JS, Milano F. Hematopoietic Stem Cell Transplantation in the Era of Engineered Cell Therapy. Curr Hematol Malig Rep. 2018;13(6):484-493.

4. Zahid U, Akbar F, Amaraneni A, Husnain M, Chan O, Riaz IB, McBride A, et al. A review of autologous stem cell transplantation in lymphoma. Curr Hematol Malig Rep. 2017;12(3):217-226.

5. Fedele R, Martino M, Recchia AG, Irrera G, Gentile M, Morabito F. Clinical options in relapsed or refractory Hodgkin lymphoma: an updated review. J Immunol Res. 2015;2015:968212.

6. Gragert L, Eapen M, Williams E, Freeman J, Spellman S, Baitty R, Hartzman R, et al. HLA match likelihoods for hematopoietic stem-cell grafts in the U.S. registry. N Engl J Med. 2014;371(4):339-348. 
7. Solomon SR, Sizemore CA, Sanacore M, Zhang X, Brown S, Holland HK, Morris LE, et al. Haploidentical transplantation using $\mathrm{T}$ cell replete peripheral blood stem cells and myeloablative conditioning in patients with high-risk hematologic malignancies who lack conventional donors is well tolerated and produces excellent relapse-free survival: results of a prospective phase II trial. Biol Blood Marrow Transplant. 2012;18(12):1859-1866.

8. Ciurea SO, Mulanovich V, Saliba RM, Bayraktar UD, Jiang Y, Bassett R, Wang SA, et al. Improved early outcomes using a $\mathrm{T}$ cell replete graft compared with $\mathrm{T}$ cell depleted haploidentical hematopoietic stem cell transplantation. Biol Blood Marrow Transplant. 2012;18(12):1835-1844.

9. Bashey A, Zhang X, Sizemore CA, Manion K, Brown S, Holland HK, Morris LE, et al. T-cell-replete HLA-haploidentical hematopoietic transplantation for hematologic malignancies using post-transplantation cyclophosphamide results in outcomes equivalent to those of contemporaneous HLA-matched related and unrelated donor transplantation. J Clin Oncol. 2013;31(10):1310-1316.

10. Elmariah H, Kasamon YL, Zahurak M, Macfarlane KW, Tucker N, Rosner GL, Bolanos-Meade J, et al. Haploidentical bone marrow transplantation with post-transplant cyclophosphamide using non-first-degree related donors. Biol Blood Marrow Transplant. 2018;24(5):1099-1102.

11. Kanakry CG, Fuchs EJ, Luznik L. Modern approaches to HLA-haploidentical blood or marrow transplantation. Nat Rev Clin Oncol. 2016;13(1):10-24.

12. Aversa F, Tabilio A, Velardi A, Cunningham I, Terenzi A, Falzetti F, Ruggeri L, et al. Treatment of high-risk acute leukemia with T-cell-depleted stem cells from related donors with one fully mismatched HLA haplotype. N Engl J Med. 1998;339(17):1186-1193.

13. Aversa F, Terenzi A, Tabilio A, Falzetti F, Carotti A, Ballanti S, Felicini R, et al. Full haplotype-mismatched hematopoietic stem-cell transplantation: a phase II study in patients with acute leukemia at high risk of relapse. J Clin Oncol. 2005;23(15):3447-3454.

14. Luznik L, Jalla S, Engstrom LW, Iannone R, Fuchs EJ. Durable engraftment of major histocompatibility complex-incompatible cells after nonmyeloablative conditioning with fludarabine, low-dose total body irradiation, and posttransplantation cyclophosphamide. Blood. 2001;98(12):3456-3464.

15. Luznik L, Engstrom LW, Iannone R, Fuchs EJ. Posttransplantation cyclophosphamide facilitates engraftment of major histocompatibility complex-identical allogeneic marrow in mice conditioned with low-dose total body irradiation. Biol Blood Marrow Transplant. 2002;8(3):131138.

16. O'Donnell PV, Luznik L, Jones RJ, Vogelsang GB, Leffell MS, Phelps M, Rhubart P, et al. Nonmyeloablative bone marrow transplantation from partially HLA-mismatched related donors using posttransplantation cyclophosphamide. Biol Blood Marrow Transplant. 2002;8(7):377386.

17. Luznik L, O'Donnell PV, Symons HJ, Chen AR, Leffell MS, Zahurak M, Gooley TA, et al. HLA-haploidentical bone marrow transplantation for hematologic malignan- cies using nonmyeloablative conditioning and high-dose, posttransplantation cyclophosphamide. Biol Blood Marrow Transplant. 2008;14(6):641-650.

18. Berenbaum MC, Brown IN. Prolongation of Homograft Survival in Mice with Single Doses of Cyclophosphamide. Nature. 1963;200:84.

19. Santos GW, Owens AH, Jr. A Comparison of the Effects of Selected Cytotoxic Agents on Allogeneic Skin Graft Survival in Rats. Bull Johns Hopkins Hosp. 1965;116:327340.

20. Mayumi H, Himeno K, Tanaka K, Tokuda N, Fan JL, Nomoto K. Drug-induced tolerance to allografts in mice. XII. The relationships between tolerance, chimerism, and graft-versus-host disease. Transplantation. 1987;44(2):286-290.

21. Mayumi H, Umesue M, Nomoto K. Cyclophosphamideinduced immunological tolerance: an overview. Immunobiology. 1996;195(2):129-139.

22. Kanakry C, Ganguly S, Zahurak M, et al. Aldehyde dehydrogenase expression drive human regulatory $\mathrm{T}$ cell resistance to posttransplantation cyclophosphamide. Sci Transl Med. 2013;5(211):1-22.

23. Ganguly S, Ross DB, Panoskaltsis-Mortari A, Kanakry CG, Blazar BR, Levy RB, Luznik L. Donor CD4+ Foxp3+ regulatory $\mathrm{T}$ cells are necessary for posttransplantation cyclophosphamide-mediated protection against GVHD in mice. Blood. 2014;124(13):2131-2141.

24. Burroughs LM, O'Donnell PV, Sandmaier BM, Storer BE, Luznik L, Symons HJ, Jones RJ, et al. Comparison of outcomes of HLA-matched related, unrelated, or HLAhaploidentical related hematopoietic cell transplantation following nonmyeloablative conditioning for relapsed or refractory Hodgkin lymphoma. Biol Blood Marrow Transplant. 2008;14(11):1279-1287.

25. Tsai T, Goodman S, Saez R, Schiller G, Adkins D, Callander N, Wolff S, et al. Allogeneic bone marrow transplantation in patients who relapse after autologous transplantation. Bone Marrow Transplant. 1997;20(10):859-863.

26. Freytes CO, Loberiza FR, Rizzo JD, Bashey A, Bredeson CN, Cairo MS, Gale RP, et al. Myeloablative allogeneic hematopoietic stem cell transplantation in patients who experience relapse after autologous stem cell transplantation for lymphoma: a report of the International Bone Marrow Transplant Registry. Blood. 2004;104(12):37973803.

27. Radich JP, Gooley T, Sanders JE, Anasetti C, Chauncey T, Appelbaum FR. Second allogeneic transplantation after failure of first autologous transplantation. Biol Blood Marrow Transplant. 2000;6(3):272-279.

28. Anderlini P, Giralt S, Andersson B, Ueno NT, Khouri I, Acholonu S, Cohen A, et al. Allogeneic stem cell transplantation with fludarabine-based, less intensive conditioning regimens as adoptive immunotherapy in advanced Hodgkin's disease. Bone Marrow Transplant. 2000;26(6):615-620.

29. Anderlini P, Saliba R, Acholonu S, Okoroji GJ, Donato M, Giralt S, Andersson B, et al. Reduced-intensity allogeneic stem cell transplantation in relapsed and refractory Hodgkin's disease: low transplant-related mortality and 
impact of intensity of conditioning regimen. Bone Marrow Transplant. 2005;35(10):943-951.

30. Devetten M, Hari P, Carreras J, et al. Unrelated Donor Nonmyeloablative/Reduced Intensity (NST/RIC) Hematopoietic Stem Cell Transplantation (HCT) for patients with relapsed and refractory Hodgkin's Lymphoma (HL). Blood. 2016;108:601.

31. Devetten MP, Hari PN, Carreras J, Logan BR, van Besien $\mathrm{K}$, Bredeson $\mathrm{CN}$, Freytes $\mathrm{CO}$, et al. Unrelated donor reduced-intensity allogeneic hematopoietic stem cell transplantation for relapsed and refractory Hodgkin lymphoma. Biol Blood Marrow Transplant. 2009;15(1):109117.

32. Sorror ML, Maris MB, Storb R, Baron F, Sandmaier BM, Maloney DG, Storer B. Hematopoietic cell transplantation (HCT)-specific comorbidity index: a new tool for risk assessment before allogeneic HCT. Blood. 2005;106(8):2912-2919.

33. Peggs KS, Hunter A, Chopra R, Parker A, Mahendra P, Milligan D, Craddock C, et al. Clinical evidence of a graft-versus-Hodgkin's-lymphoma effect after reduced-intensity allogeneic transplantation. Lancet. 2005;365(9475):1934-1941.

34. Mariotti J, Devillier R, Bramanti S, Sarina B, Furst S, Granata A, Faucher C, et al. T Cell-replete haploidentical transplantation with post-transplantation cyclophosphamide for Hodgkin lymphoma relapsed after autologous transplantation: reduced incidence of relapse and of chronic graft-versus-host disease compared with HLAidentical related donors. Biol Blood Marrow Transplant. 2018;24(3):627-632.

35. Gauthier J, Castagna L, Garnier F, Guillaume T, Socie G, Maury S, Maillard N, et al. Reduced-intensity and non-myeloablative allogeneic stem cell transplantation from alternative HLA-mismatched donors for Hodgkin lymphoma: a study by the French Society of Bone Marrow Transplantation and Cellular Therapy. Bone Marrow Transplant. 2017;52(5):689-696.

36. Martinez C, Gayoso J, Canals C, Finel H, Peggs K, Dominietto A, Castagna L, et al. Post-transplantation cyclophosphamide-based haploidentical transplantation as alternative to matched sibling or unrelated donor transplantation for Hodgkin lymphoma: a registry study of the lymphoma working party of the European society for blood and marrow transplantation. J Clin Oncol. 2017;35(30):3425-3432.

37. Raiola A, Dominietto A, Varaldo R, Ghiso A, Galaverna F, Bramanti S, Todisco E, et al. Unmanipulated haploidentical BMT following non-myeloablative conditioning and post-transplantation CY for advanced Hodgkin's lymphoma. Bone Marrow Transplant. 2014;49(2):190-194.

38. Gayoso J, Balsalobre P, Pascual MJ, Castilla-Llorente C, Lopez-Corral L, Kwon M, Serrano D, et al. Busulfanbased reduced intensity conditioning regimens for haploidentical transplantation in relapsed/refractory Hodgkin lymphoma: Spanish multicenter experience. Bone Marrow Transplant. 2016;51(10):1307-1312.

39. Marani C, Raiola AM, Morbelli S, Dominietto A, Ferrarazzo G, Avenoso D, Giannoni L, et al. Haploidenti- cal transplants with post-transplant cyclophosphamide for relapsed or refractory Hodgkin lymphoma: the role of comorbidity index and pretransplant positron emission tomography. Biol Blood Marrow Transplant. 2018;24(12):2501-2508.

40. Lacerda MP, Arrais Rodrigues C, Pereira AD, Novis Y, Fonseca M, Silva RL, Macedo M, et al. Human Leukocyte Antigen-Haploidentical Transplantation for Relapsed/Refractory Hodgkin Lymphoma: A Multicenter Analysis. Biol Blood Marrow Transplant. 2017;23(4):705-707.

41. Castagna L, Bramanti S, Devillier R, Sarina B, Crocchiolo R, Furst S, El-Cheikh J, et al. Haploidentical transplantation with post-infusion cyclophosphamide in advanced Hodgkin lymphoma. Bone Marrow Transplant. 2017;52(5):683-688.

42. Castagna L, Bramanti S, Furst S, Giordano L, Crocchiolo R, Sarina B, Mauro E, et al. Nonmyeloablative conditioning, unmanipulated haploidentical SCT and post-infusion CY for advanced lymphomas. Bone Marrow Transplant. 2014;49(12):1475-1480.

43. Kanate AS, Mussetti A, Kharfan-Dabaja MA, Ahn KW, DiGilio A, Beitinjaneh A, Chhabra S, et al. Reduced-intensity transplantation for lymphomas using haploidentical related donors vs HLA-matched unrelated donors. Blood. 2016;127(7):938-947.

44. Ghosh N, Karmali R, Rocha V, Ahn KW, DiGilio A, Hari $\mathrm{PN}$, Bachanova $\mathrm{V}$, et al. Reduced-intensity transplantation for lymphomas using haploidentical related donors versus HLA-matched sibling donors: a center for international blood and marrow transplant research analysis. J Clin Oncol. 2016;34(26):3141-3149.

45. Dietrich S, Finel H, Martinez C, Tischer J, Blaise D, Chevallier P, Castagna L, et al. Post-transplant cyclophosphamide-based haplo-identical transplantation as alternative to matched sibling or unrelated donor transplantation for non-Hodgkin lymphoma: a registry study by the European society for blood and marrow transplantation. Leukemia. 2016;30(10):2086-2089.

46. Ruggeri A, Labopin M, Bacigalupo A, Gulbas Z, Koc Y, Blaise D, Bruno B, et al. Bone marrow versus mobilized peripheral blood stem cells in haploidentical transplants using posttransplantation cyclophosphamide. Cancer. 2018;124(7):1428-1437.

47. Chen R, Palmer JM, Thomas SH, Tsai NC, Farol L, Nademanee A, Forman SJ, et al. Brentuximab vedotin enables successful reduced-intensity allogeneic hematopoietic cell transplantation in patients with relapsed or refractory Hodgkin lymphoma. Blood. 2012;119(26):6379-6381.

48. Chen R, Gopal AK, Smith SE, Ansell SM, Rosenblatt JD, Savage KJ, Connors JM, et al. Five-year survival and durability results of brentuximab vedotin in patients with relapsed or refractory Hodgkin lymphoma. Blood. 2016;128(12):1562-1566.

49. Chen R, Zinzani PL, Fanale MA, Armand P, Johnson NA, Brice P, Radford J, et al. Phase II study of the efficacy and safety of pembrolizumab for relapsed/refractory classic Hodgkin lymphoma. J Clin Oncol. 2017;35(19):21252132.

50. Ansell SM, Lesokhin AM, Borrello I, Halwani A, Scott 
EC, Gutierrez M, Schuster SJ, et al. PD-1 blockade with nivolumab in relapsed or refractory Hodgkin's lymphoma. N Engl J Med. 2015;372(4):311-319.

51. Merryman RW, Kim HT, Zinzani PL, Carlo-Stella C, Ansell SM, Perales MA, Avigdor A, et al. Safety and efficacy of allogeneic hematopoietic stem cell transplant after PD-1 blockade in relapsed/refractory lymphoma. Blood. 2017;129(10):1380-1388.

52. Wanquet A, Bramanti S, Harbi S, Furst S, Legrand F, Faucher C, Granata A, et al. Killer cell immunoglobulin-like receptor-ligand mismatch in donor versus recipient direction provides better graft-versus-tumor effect in patients with hematologic malignancies undergoing allogeneic $\mathrm{T}$ cell-replete haploidentical transplantation followed by post-transplant cyclophosphamide. Biol Blood Marrow Transplant. 2018;24(3):549-554.

53. Ruggeri L, Mancusi A, Burchielli E, Capanni M, Carotti
A, Aloisi T, Aversa F, et al. NK cell alloreactivity and allogeneic hematopoietic stem cell transplantation. Blood Cells Mol Dis. 2008;40(1):84-90.

54. Russo A, Oliveira G, Berglund S, Greco R, Gambacorta $\mathrm{V}$, Cieri N, Toffalori $\mathrm{C}$, et al. NK cell recovery after haploidentical HSCT with posttransplant cyclophosphamide: dynamics and clinical implications. Blood. 2018;131(2):247-262.

55. Aversa F, Bachar-Lustig E, Or-Geva N, Prezioso L, Bonomini S, Manfra I, Monti A, et al. Immune tolerance induction by nonmyeloablative haploidentical HSCT combining T-cell depletion and posttransplant cyclophosphamide. Blood Adv. 2017;1(24):2166-2175.

56. Liu J, Zhong JF, Zhang X, Zhang C. Allogeneic CD19CAR-T cell infusion after allogeneic hematopoietic stem cell transplantation in B cell malignancies. J Hematol Oncol. 2017;10(1):35. 